\title{
Analysis of the Winning Rate of Asymmetric Antagonistic Games
}

\author{
Li Yan-Bin ${ }^{1}$, Zhang Hu-Ling ${ }^{2}$ \\ ${ }^{1}$ Department of Electrical Engineering, University of Sydeny, Sydeny, Australia \\ ${ }^{2}$ JiangYin TianRen Industry Technological Service Co., LTD, Jiangyin, China
}

\section{Email address:}

2653675198@qq.com (Li Yan-Bin), trgyjsfw@163.com (Zhang Hu-Ling)

\section{To cite this article:}

Li Yan-Bin, Zhang Hu-Ling. Analysis of the Winning Rate of Asymmetric Antagonistic Games. Science Discovery. Vol. 7, No. 4, 2019, pp. 205-208. doi: 10.11648/j.sd.20190704.15

Received: July 6, 2019; Accepted: July 24, 2019; Published: July 29, 2019

\begin{abstract}
Game theory is a mathematical theory and method for studying phenomena with the nature of struggle, it is one of the most influential theories in modern social sciences. The idea of game theory is very well embodied in some asymmetric confrontation games. Players decision also count a lot in winning the game, but designers should balance the game by who should occupy the offensive as well as their abilities. This passage simply discusses the construction and the settlement of the simplest model of them - general three-man duel model, then gives out a way to solve the problem of finding the survival rate of any duelist after several given rounds of the game. This method can be extended to general n-man duel model. People who participate in the game usually maximize their own interests through certain rules, This study will provide reference value for the design of rules and the rational decision-making in Game Process.
\end{abstract}

Keywords: Game Theory, Asymmertric Countermeasure Games, General Three-man Duel Model, Probability, Markov Chain

\section{非对称对抗博竕的胜率分析}

\section{李延滨 ${ }^{1}$, 张沪玲 ${ }^{*}$}

1悉尼大学电气工程系, 悉尼, 澳大利亚

2江阴天人工业技术服务有限公司, 江阴, 中国

\section{邮箱}

2653675198@qq.com(李延滨), trgyjsfw@163.com（张沪玲）

摘要: 博亦论是研究具有斗争或竞争性质现象的数学理论和方法, 是对现代社会科学影响最广泛的理论之一。博亦论 的理念在一些非对称对抗游戏中有非常好的体现, 比如游戏设计者需要解决的是如何平衡先手和后手以及各个玩家操 控角色的异能强度问题; 而玩家需要根据局势进行合理的决策, 以增加其胜利的可能性。本文从最简单的“一般三人决 斗”问题入手, 进行了数学建模和行动策略分析, 使用马尔科夫链给出了求解各种情况下任意轮之后各个决斗者生存概 率的一种计算方法, 该方法可以拓展到“一般 $\mathrm{n}$ 人决斗”问题。参与博弯的人通常会通过一定的规则使自己的利益最大化, 本文的研究会对规则的设计和博弯过程中的合理决策提供参考价值。

关键字：博弯论，非对称对抗游戏，一般三人决斗，概率，马尔科夫链 


\section{1. 引言}

在二战后的 50 年里对社会科学影响最广泛的是什么 理论, 不同领域的人可能会有不同的回答, 而大多数数学 家尤其是经济学家会认为, 对社会科学影响最广泛的理论 应该是博亦论 (Game theory)。博亦论主要研究公式化了 的激励结构间的相互作用, 是研究具有斗争或竞争性质现 象的数学理论和方法 [1]。博亦论考虑游戏中的个体的预测 行为和实际行为, 并研究它们的优化策略。

我们以游戏为例: 多人回合制非对称对抗游戏中，在 游戏的设计过程中, 设计者需要解决如何平衡先手和后手 以及各个玩家操控角色的异能强度问题。一般而言, 异能 效果设计弱的角色在游戏设计时往往会被给与游戏内资 源上的优势或者是行动顺序上的补正, 以达到调整各角色 胜率, 平衡游戏的目的。而玩家在玩游戏的过程中, 则需 要考虑如何进行自身的行动, 也需要根据局势进行合理的 决策, 以增加其胜利的可能性。

美国纽约大学政治学系著名的政治学家勃拉姆斯做 的“3人决斗模型”游戏, 在政治学中就有广泛影响 $[2]$ 。本 文从最简单的三人决斗问题入手讨论, 进行了简单的数学 建模和行动策略分析, 进而类推到多人回合制非对称对抗 游戏中。

\section{2. 一般三人决斗问题中各个决斗者的策略分析 \\ 2.1. 一般三人决斗问题}

经典的三人决斗问题, 三个决斗者依照枪法由差至 好, 依次轮流向另一名决斗者射击, 直至最后只剩一名决 斗者。

设定每个决斗者都可以任意选择射击对象, 也可以选 择放空枪, 但是此时存活的枪法最好的决斗者不得放空 枪; 设定任何决斗者一旦被击中则判定为死亡, 无法继续 射击或者被射击。

基于以上设定, 本文分析了每个决斗者的射击策略, 并求出他们最后生还的概率。已知任意决斗者只要不选择 放空枪让过, 则不论选择谁作为射击对象, 其命中率始终 为一个已知的常数。

\section{2. 一般三人决斗先手优势}

假定两个人 $\mathrm{X}, \mathrm{Y}$, 两个人由其中一个开始轮流向对 方射击。假定 $X$ 的命中率为 $\mathrm{x}, \mathrm{Y}$ 命中率为 $\mathrm{y}[3-4]$ 。

那么, $\mathrm{X}$ 先手时的存活率为:

$$
P_{X Y}=\frac{x}{x+y-x y}
$$

$\mathrm{X}$ 后手时的存活率为:

$$
P_{Y X=} \frac{x}{x+y-x y} \frac{x-x y}{x+y-x y}
$$

由于 $x 、 y$ 均大于 0 , 故有:

$$
\frac{x}{x+y-x y}>\frac{x}{y}
$$

可见先手攻击能一定程度上提高存活率。 如果有一人 $M$, 其命中率为 $m$, 且有:

$$
\mathrm{x}<\mathrm{m}<\mathrm{y}
$$

并且给予X如下选择:

与 $Y$ 决斗, 且 $X$ 先手;

(2) 与 $M$ 决斗, 且 $M$ 先手;

那么当:

$$
\frac{x}{x+y-x y}>\frac{x}{x+m-x m} \cdot \frac{x-x m}{x+m-x m}
$$

或者:

$$
\frac{x}{x+y-x y}+\frac{x}{x+m-x m}>1
$$

时, X选择(1)的生存概率更大, 也即此时先手优势相 比于选择命中率更低的对手而言更大。

\section{3. 三人决斗问题中各个决斗者的策略分析}

假设三个人分别为 $\mathrm{A} 1, \mathrm{~A} 2, \mathrm{~A} 3$, 其命中率由低至高 排列, 且分别为 $a 1 、 a 2 、 a 3$; 由 $A 1$ 先开枪。

(1) A3的射击策略为: 先射击A2直到命中, 再射击 A1。

(2) 对于A2而言:

假定A1已经死亡, 情况则变为 $\mathrm{A} 2 、 \mathrm{~A} 3$ 两人决斗; 假定 $\mathrm{A} 1$ 已经死亡, 情况则变为 $\mathrm{A} 2 、 \mathrm{~A} 3$ 两人决斗; 假定 $\mathrm{A} 1, \mathrm{~A} 3$ 均存活, 那么A2会射击 $\mathrm{A} 3$ ，

原因如下:

此时 $\mathrm{A} 1 、 \mathrm{~A} 2$ 都可以选择射击或者让过。由于 $\mathrm{A} 3$ 威胁 最大, 所以假定任何一人选择射击, 则必定会选择射击 $\mathrm{A} 3$ 。 共四种情况如下:

$\mathrm{A} 1$ 让过, $\mathrm{A} 2$ 射击 $\mathrm{A} 3, \mathrm{~A} 3$ 射击 $\mathrm{A} 2$;

$\mathrm{A} 1$ 让过, $\mathrm{A} 2$ 让过, $\mathrm{A} 3$ 射击 $\mathrm{A} 2$;

$A 1$ 射击 $A 3, A 2$ 射击 $A 3, A 3$ 射击 $A 2$;

A1射击 $A 3, A 2$ 让过 $A 3, A 3$ 射击 $A 2$;

对比情况 $1 、 2$ 可知, 假定 $\mathrm{A} 1$ 选择让过, 则在 $\mathrm{A} 2 、 \mathrm{~A} 3$ 一个人死亡前, 情况实际转化为 $\mathrm{A} 2 、 \mathrm{~A} 3$ 两人决斗, 因此 A2此时会选择射击A3;

对比情况 $3 、 4$ 可知, 假定 $\mathrm{A} 2$ 射击命中 $\mathrm{A} 3$, 那么将变 成 $A 1 、 A 2$ 两人决斗且 $A 1$ 先手, 也可以视为在 $A 1$ 射击 $A 2$ 后进行由 $\mathrm{A} 2$ 先手的 $\mathrm{A} 1 、 \mathrm{~A} 2$ 两人决斗; 假定 $\mathrm{A} 2$ 未命中或者 让过, 那么 $\mathrm{A} 3$ 将继续射击 $\mathrm{A} 2$ 。接下来情况又分为:

A1命中 A3;

A1未命中A3;

情况 1 下, $\mathrm{A} 2$ 的威胁为: $\mathrm{A} 3$ 射击 $\mathrm{A} 2$ 后进行由 $\mathrm{A} 2$ 先手 的 $\mathrm{A} 1 、 \mathrm{~A} 2$ 两人决斗。又由于 $\mathrm{a} 3>\mathrm{a} 1$, 故此时 $\mathrm{A} 2$ 首先射击命 中 $\mathrm{A} 3$ 的生存率大于 $\mathrm{A} 2$ 未命中、让过, 且 $\mathrm{A} 1$ 命中 $\mathrm{A} 3$ 时的生 
存率。若A1未命中则重复如上流程, 每一次A2、A1未命 中则 $\mathrm{A} 3$ 将增加一轮对 $\mathrm{A} 2$ 的射击。

A1

综上, A2策略为: 先射击 $\mathrm{A} 3$, 如果 $\mathrm{A} 3$ 死亡则再射击

（3）对于 $\mathrm{A} 1$ 而言: 其优势策略有两种: 射击 $\mathrm{A} 3$, 或 者放空枪。具体选择哪种策略取决于所有人的命中率 ${ }^{[2]}$ 。 由式 (1)、（2）可知A1在不同情况下的存活率如下 [5-7]:

A1对A2, A1先手的存活率:

$$
\mathrm{P}(1 ; 1,2 ; 1)=\frac{\mathrm{a} 1}{\mathrm{a} 1+\mathrm{a} 2-\mathrm{a} 1 * \mathrm{a} 2}
$$

A1对A3, A1先手的存活率:

$$
\mathrm{P}(1 ; 1,3 ; 1)=\frac{\mathrm{a} 1}{\mathrm{a} 1+\mathrm{a} 3-\mathrm{a} 1 * \mathrm{a} 3}
$$

A1对A2, A2先手的存活率:

$$
\mathrm{P}(1 ; 1,2 ; 2)=\frac{\mathrm{a} 1-\mathrm{a} 1 * \mathrm{a} 2}{\mathrm{a} 1+\mathrm{a} 2-\mathrm{a} 1 * \mathrm{a} 2}
$$

A1对A3，A3先手的存活率:

$$
\mathrm{P}(1 ; 1,3 ; 3)=\frac{\mathrm{a} 1-\mathrm{a} 1 * \mathrm{a} 3}{\mathrm{a} 1+\mathrm{a} 3-\mathrm{a} 1 * \mathrm{a} 3}
$$

假若 $\mathrm{A} 1$ 放空枪, 那么由 $\mathrm{A} 2$ 和 $\mathrm{A} 3$ 决斗的结果为（此时 A2先手) :

A2命中 $\mathrm{A} 3$ 的概率:

$$
\begin{aligned}
& \Delta \mathrm{P}=[\mathrm{P} 2-\mathrm{P}(2 ; 2,3 ; 2)] * \mathrm{P}(1 ; 1,2 ; 1)+[\mathrm{P} 3-\mathrm{P}(3 ; 2,3 ; 2)] * \mathrm{P}(1 ; 1,3 ; 1) \\
& +\mathrm{P} 1 * \mathrm{P}(1 ; 1,2 ; 2) \\
& =\left[\begin{array}{c}
-\mathrm{a} 1 * \mathrm{a} 2 * \frac{\mathrm{a} 1}{\mathrm{a} 1+\mathrm{a} 2-\mathrm{a} 1 * \mathrm{a} 2}-(\mathrm{a} 1 * \mathrm{a} 2 * \mathrm{a} 3-\mathrm{a} 1 * \mathrm{a} 3) * \frac{\mathrm{a} 1}{\mathrm{a} 1+\mathrm{a} 3-\mathrm{a} 1 * \mathrm{a} 3} \\
+\mathrm{a} 1 *(\mathrm{a} 2+\mathrm{a} 3-\mathrm{a} 2 * \mathrm{a} 3) * \frac{\mathrm{a} 1-\mathrm{a} 1 * \mathrm{a} 2}{\mathrm{a} 1+\mathrm{a} 2-\mathrm{a} 1 * \mathrm{a} 2}
\end{array}\right] * \frac{1}{\mathrm{k}} \\
& \text { （其中 } \mathrm{k}=(\mathrm{a} 1+\mathrm{a} 2+\mathrm{a} 3-\mathrm{a} 1 * \mathrm{a} 2-\mathrm{a} 1 * \mathrm{a} 3-\mathrm{a} 2 * \mathrm{a} 3+\mathrm{a} 1 * \mathrm{a} 2 * \mathrm{a} 3) \\
& *(\mathrm{a} 2+\mathrm{a} 3-\mathrm{a} 2 * \mathrm{a} 3) \text {, 必定是一个正数。) } \\
& =\left[\begin{array}{c}
-\mathrm{a} 1^{2} * \mathrm{a} 2 *(\mathrm{a} 1+\mathrm{a} 3-\mathrm{a} 1 * \mathrm{a} 3)-\mathrm{a} 1 *(\mathrm{a} 1 * \mathrm{a} 2 * \mathrm{a} 3-\mathrm{a} 1 * \mathrm{a} 3) \\
*(\mathrm{a} 1+\mathrm{a} 2-\mathrm{a} 1 * \mathrm{a} 2)+\mathrm{a} 1 *(\mathrm{a} 2+\mathrm{a} 3-\mathrm{a} 2 * \mathrm{a} 3) *(\mathrm{a} 1+\mathrm{a} 3-\mathrm{a} 1 * \mathrm{a} 3)
\end{array}\right] \\
& * \frac{1}{\mathrm{k}} * \frac{1}{\mathrm{p}} \\
& \text { (其中 } \mathrm{p}=(\mathrm{a} 1+\mathrm{a} 2-\mathrm{a} 1 * \mathrm{a} 2) *(\mathrm{a} 1+\mathrm{a} 3-\mathrm{a} 1 * \mathrm{a} 3) \text {, 必定为正数。) } \\
& =\left[\begin{array}{c}
\mathrm{a} 1^{3} *\left(-\mathrm{a} 2-\mathrm{a} 2^{2} * \mathrm{a} 3+\mathrm{a} 3\right) \\
+\mathrm{a} 1^{2} *\left(-\mathrm{a} 2^{2} * \mathrm{a} 3+\mathrm{a} 2 * \mathrm{a} 3^{2}-3 \mathrm{a} 2 * \mathrm{a} 3-\mathrm{a} 3^{2}+\mathrm{a} 2+\mathrm{a} 3\right) \\
+\mathrm{a} 1 *\left(\mathrm{a} 2 * \mathrm{a} 3-\mathrm{a} 2 * \mathrm{a} 3^{2}\right)
\end{array}\right] * \frac{1}{\mathrm{k} * \mathrm{p}}
\end{aligned}
$$

因此, 当上式方括号内数值为正时, A1会选择射击 $\mathrm{A} 3$, 否则 $\mathrm{A} 1$ 会开空枪直到 $\mathrm{A} 2, \mathrm{~A} 3$ 其中一人被命中。

\section{3. 一般 $\mathrm{n}$ 人决斗各个决斗者的策略分析}

\section{1. 使用马尔科夫链进行各个决斗者生存概率的计算}

定义从一名决斗决斗者开始, 各个决斗者依次进行一 次射击直至枪法最好者射击结束为一轮射击。
对于每个决斗者而言, 在每一轮射击开始前, 其存在 状态可以用存活或者死亡来表示。假若用 1 来表示该名决 斗者存活, 用 0 来表示该名决斗者死亡, 那么当决斗者的 人数为 $\mathrm{N}$ 时, 所有可能存在的决斗者存活、死亡的状态排 列一共有 $2 n-1$ 种（全部死亡的情况除外）。对于 3 人决斗 问题, 所有可能的状态有:
A1
A2
A3
1 


$\begin{array}{lll}1 & 1 & 0 \\ 1 & 0 & 1 \\ 1 & 0 & 0 \\ 0 & 1 & 1 \\ 0 & 1 & 0 \\ 0 & 0 & 1\end{array}$

并且这些状态不能共存。因此, 可以建立一个 $1 *(2 n-1)$ 的列矩阵X来描述在任意一轮开始时各个状态存在的概率。

另外, 对于每一名决斗者而言, 其射击策略的选择仅 与当前各个决斗者的存活状态有关, 因此每一轮射击过后 系统状态的转变仅是这一轮射击前系统状态的函数。因此 可以建立一个 $7 * 7$ 的转移矩阵 $\mathrm{A}[8-10]$, 用来表示每一轮射 击后各个决斗者的生存概率。假定经历过 $\mathrm{n}$ 轮射击后, 系 统状态为 $Y$, 则有:

\section{$\mathrm{A}^{\mathrm{n}} \mathrm{X}=\mathrm{Y}$}

(上式中 $\mathbf{A}$ 为转移矩阵, $\mathbf{X}$ 为初始状态矩阵, $\mathrm{Y}$ 为 $\mathrm{n}$ 轮 之后的解矩阵。)

转移矩阵 $\mathbf{A}=\left[\begin{array}{ccc}\mathrm{a} 111,111 & \cdots & \mathrm{a} 111,001 \\ \vdots & \ddots & \vdots \\ \mathrm{a} 001,111 & \cdots & \mathrm{a} 001,001\end{array}\right]$;

其中 $a_{P}, Q$ 表示经历一轮射击, 系统从 $P$ 状态转换为 $Q$ 状 态的概率。

由于初始时所有人存活, 故有: $\mathbf{X}=\left[\begin{array}{lllll}1 & 0 & 0 & \cdots & 0\end{array}\right]^{T}$;

$\mathbf{Y}$ 矩阵的求解可以由特征向量法进行求解, 在此不多 赘述。

每个人在 $\mathrm{n}$ 轮之后的生存概率可以由使得解矩阵元素 下标对应的情况中该人存活的情况进行求和运算得到。举 例来讲, 要计算 $\mathrm{A} 1$ 在 $\mathrm{n}$ 轮之后的存活率, 只需将 $\mathbf{Y}$ 中的元 素 $\mathrm{a}_{111}, \mathrm{a}_{110}, \mathrm{a}_{101}, \mathrm{a}_{100}$ 进行求和即可。

当 $\mathrm{n}$ 趋向于无穷大时, 解矩阵的极限为: $\mathrm{Y}=\left[\begin{array}{lllllll}0 & 0 & \cdots & \mathrm{P}_{\mathrm{A} 1} & \cdots & \mathrm{P}_{\mathrm{A} 2} & \mathrm{P}_{\mathrm{A} 3}\end{array}\right]^{T}$,

其中 $\mathrm{P}_{\mathrm{A} 1}, \mathrm{P}_{\mathrm{A} 2}, \mathrm{P}_{\mathrm{A} 3}$ 分别为 $\mathrm{A} 1, \mathrm{~A} 2, \mathrm{~A} 3$ 的最终生还的 概率。

\section{2. $\mathrm{n}$ 人决斗各个决斗者的策略分析}

当问题变为“一般n人决斗”时，假定这些人依照枪法 由差到好, 由左至右站成一排。对于每个人, 其威胁来自 两部分：第一是比自己命中率高的一侧（称为“右侧”）的 不断逼近的压力; 另一部分是比自己命中率低的一侧 (称 为“左侧”）的先手优势。这两种威胁, 实际上是由于自身 在射击序列中的位置所决定的。因此这个人要么不射击, 要么就会清理右侧威胁, 要么就会清理左侧威胁。清理右 侧威胁的最佳手段是射击现存的枪法最好的人; 清理左侧 威胁的最好手段就是射击自己左侧最靠近自己的人。

\section{4. 结论}

对于非对称对抗游戏而言, 角色数值的调整关系着该 角色的威胁程度的变化, 进而影响所有其他玩家的策略以
及生存率。同时, 由于强力角色的威胁大, 其胜率在某些 情况下甚至会低于其他较弱角色。在非对称对抗游戏中, 最强者会依照威胁由大至小逐步去除威胁, 次强者会对抗 最强者, 中等程度者的决策较复杂, 需要具体分析, 次弱 者会清除最弱者, 最弱者会尝试击败最强者。

现实中，很多个体间的关系，都是非对称的，基于利 益关系, 有时候也是具有一定对抗性质的, 而合作很多时 候是建立在利益分享上的。参与博弯的人通常会通过一定 的规则使自己的利益最大化, 本文的研究的结论会对规则 的设计和博亦过程中的决策提供参考价值。

\section{参考文献}

[1] 博弯论及其应用 $[\mathrm{M}]$, 杭州：浙江大学出版社, 2015.09。

[2] 动态合作博亦[M], 科学出版社, 2009。

[3] 数学 (苏教版) 选修二[M], 江苏凤凰教育出版社, 2015。

[4] (苏) 温特切勒, 概率论 $[\mathrm{M}]$, 科学技术出版社, 1961。

[5] 概率论与随机过程 $[\mathrm{M}]$, 清华大学出版社, 2013.08。

[6] 随机过程导论[M], 机械工业出版社, 2010.09。

[7] 概率论与数理统计 $[\mathrm{M}]$, 高等教育出版社, 2014.12。

[8] 施仁杰, 马尔科夫链基础及其应用 $[\mathrm{M}]$, 西安: 西安电子科 技大学出版社, 1992.11。

[9] 杨明刘先忠, 矩阵论 $[\mathrm{M}]$, 武汉: 华中科技大学出版社。

[10] 生灭过程与马尔科夫链 (第二版) [M], 科学出版社, 2005.01。

\section{作者简介}

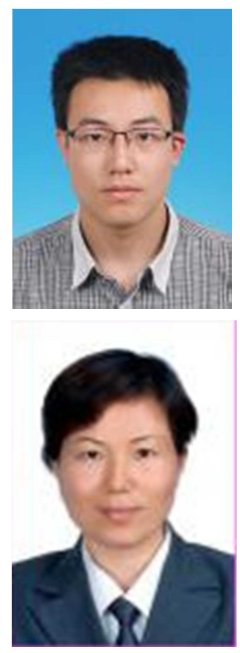

李延滨（1998-）, 男, 江苏江阴人, 悉 尼大学本科在读, 电气工程专业。

张沪玲(1971-)，女，陕西华县人，国防 科学技术大学通信与网络硕士, 高级工 程师, 主要研究方向为网络安全。 